\section{Outgrowing IRAK-4}

Humans outgrow their need for a TLR-activated kinase, according to a new study by $\mathrm{Ku}$ et al. (page 2407). The kinase helps protect young children from specific pathogens but is expendable in adults.

The TLRs are part of an early infection warning system that recognizes microbial intrusion. Many activated TLRs recruit a kinase called IRAK-4, which switches on immune-boosting transcription pathways.

In mice, IRAK-4 is thought to be a crucial cog in innate defense, as mice of all ages lacking the kinase are vulnerable to many different viruses, bacteria, and fungi. But the human version now appears to be less comprehensive.

The new study examined 28 patients with IRAK-4 mutations. Immune cells from these patients' blood failed to produce inflammatory cytokines in response to TLR ligation. During infancy and childhood, these patients experienced repeated, sometimes lethal infections mainly with Streptococcus pneumoniae and Staphylococcus aureus. Resistance to other pathogens might stem from TLRs that bypass IRAK-4, including possibly TLR3 and TLR4, or from nonTLR pathways.

About half of the young children succumbed to the bacterial infections. But the patients' resistance improved with age, and older children consistently survived infections. The impact of IRAK-4 deficiency might fade as the adaptive immune network, particularly memory responses by $\mathrm{T}$ and $\mathrm{B}$ cells, kicks in later in life.

It is unclear why IRAK-4 deficiency preferentially opens the door for some invasive bacteria but not other pathogens. The team has previously identified defects in other innate immunity factors that render patients susceptible to just one pathogen. A mechanistic explanation for this selectivity is yet to be found. JEM

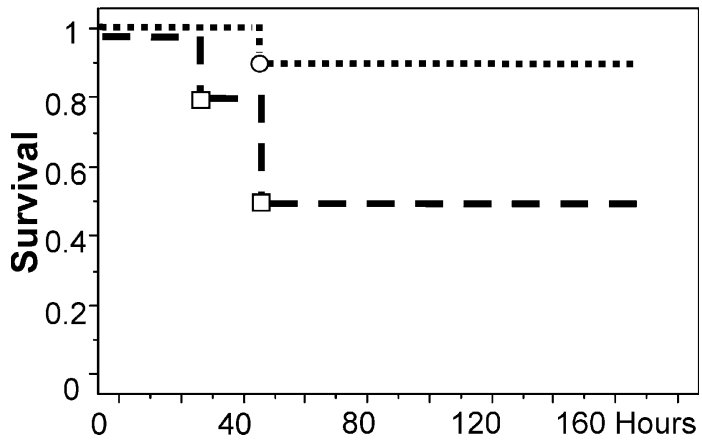

Mice are protected from sepsis-induced death by an APC variant that lacks its anticlotting activity (circle).

\section{Improving survival after sepsis}

The only drug that can save patients from severe sepsis carries a risk of internal bleeding. Kerschen et al. (page 2439) have now engineered a variant version that offers the same benefits as the original but minimizes the risk.

Sepsis is a system-wide inflammation that occurs when an infecting microbe enters the bloodstream. The inflammation is meant to fight the pathogen, but it also triggers widespread clotting. Clotting proteins, in turn, reinforce inflammation by activating oxidants and proteases, ultimately causing organ failure. Because the exact cause of death in sepsis patients is unknown, scientists don't know what problem to target when designing an effective therapy.

Antiinflammatory agents such as steroids and cytokine antagonists have failed as therapies. So too have anticlotting agents. The only treatment that has had any degree of success is activated protein C (APC).

APC is both a clot-busting enzyme and a signaling molecule that blocks apoptosis, inflammatory cytokine production, and immune cell recruitment. APC is therefore thought to work against sepsis by breaking the inflammation/clotting cycle. But patients dosed with APC can experience internal bleeding thanks to the drug's anticlotting activity.

Kerschen and colleagues now test whether APC might still be effective but less dangerous if its anticlotting activity is reduced. They engineered an APC variant that was $90 \%$ less effective at stopping clotting. This variant was just as good as normal APC at saving septic mice, suggesting that the protective effect of APC did not stem from its anticlotting function but from its signaling ability.

Exactly why APC's antiinflammatory signaling activity succeeds in treating sepsis where other antiinflammatory drugs have failed is unclear, but this might be due to its broader effects on both the inflammatory and apoptotic pathways. JEM 